\title{
A systematic review of the adoption and acceptance of eHealth in Saudi Arabia: views of multiple stakeholders.
}

ALSHAHRANI, A., STEWART, D. and MACLURE, K. 


\section{Accepted Manuscript}

Title: A systematic review of the adoption and acceptance of eHealth in Saudi Arabia: views of multiple stakeholders

Authors: Abdullah Alshahrani, Derek Stewart, Katie MacLure

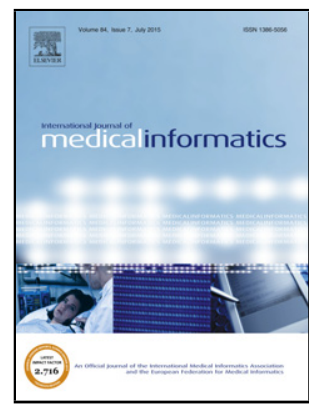

PII:

S1386-5056(18)30722-6

DOI: https://doi.org/10.1016/j.ijmedinf.2019.05.007

Reference: IJB 3871

To appear in: International Journal of Medical Informatics

Received date: $\quad 28$ June 2018

Revised date: $\quad 23$ February 2019

Accepted date: $\quad 9$ May 2019

Please cite this article as: Alshahrani A, Stewart D, MacLure K, A systematic review of the adoption and acceptance of eHealth in Saudi Arabia: views of multiple stakeholders, International Journal of Medical Informatics (2019), https://doi.org/10.1016/j.ijmedinf.2019.05.007

This is a PDF file of an unedited manuscript that has been accepted for publication. As a service to our customers we are providing this early version of the manuscript. The manuscript will undergo copyediting, typesetting, and review of the resulting proof before it is published in its final form. Please note that during the production process errors may be discovered which could affect the content, and all legal disclaimers that apply to the journal pertain. 
A systematic review of the adoption and acceptance of eHealth in Saudi Arabia: views of multiple stakeholders

Abdullah Alshahrani (lead author) ${ }^{1}$, Professor Derek Stewart ${ }^{1}$, Dr Katie MacLure ${ }^{1, *}$

${ }^{1}$ School of Pharmacy and Life Sciences, Robert Gordon University, Aberdeen, UK

* Corresponding author:

Dr Katie MacLure

School of Pharmacy \& Life Sciences

Robert Gordon University

Sir Ian Wood Building, Garthdee Road, Aberdeen UK, AB10 7GJ

Email: K.M.MacLure@rgu.ac.uk 


\section{Abstract}

Background: eHealth is defined as "the use of information and communication technology for health". Adoption and acceptance are key concepts to measure the level of eHealth impact. The aim of this systematic review was to critically appraise, synthesise and present evidence of the status of eHealth adoption and acceptance in Saudi Arabia from the perspectives of multiple stakeholders.

Methods: Based on a Preferred Reporting Items for Systematic review and Meta-Analysis Protocols (PRISMA-P) guided protocol published with the international prospective register of systematic reviews (Prospero), five databases were searched for articles published between 1993 and 2017. Inclusion and exclusion criteria of studies were applied in which only peer-reviewed, full-text primary research articles in English language were included. One reviewer performed the searches; two reviewers independently screened the titles then abstracts followed by full articles. Studies excluded were recorded with reasons. Critical appraisal tools appropriate to study design were applied. Eleven items from every study were extracted for further synthesis.

Results: After duplicates were removed, 110 papers were screened, and 15 studies met the inclusion criteria. Studies were generally of good quality. Thirty-nine factors were identified as influences affecting the adoption and acceptance of eHealth in Saudi Arabia. Lack of eHealth studies from the perspective of health managers and the limitation of studies to few geographical areas were identified as knowledge gaps.

Conclusion: eHealth field in Saudi Arabia showed evidence of continual growth in both publications and awareness of significance. Therefore, findings from this review may help key professionals to address the current challenges and barriers and prioritise the main areas for improvement.

Keywords eHealth; telemedicine; Electronic Health Record; Electronic Medical record; acceptance; adoption; Saudi Arabia 


\section{Introduction}

The evolution of the use of 'e'-terminology started in the 1990s, embracing the global advancement in the field of Information and Communication Technology (ICT). For example, electronic mail or email made it possible for people to communicate rapidly, ecommerce invented ways for conducting business and finance, and eHealth is utilised for improving the outcomes of healthcare systems [1]. The term eHealth came into use in the year 2000, but has since become wide spread globally [2]. The definition of this term was focused on from different perspectives. The World Health Organization (WHO) defined it as "the use of ICT for health". This definition has encompassed two main concepts (health and technology) in a broadly unique concept (eHealth) [3]. Pretlow (2000) defined it based on the use of internet "the process of providing health care via electronic means, in particular over the Internet. It can include teaching, monitoring, and interaction with health care providers, as well as interaction with other patients afflicted with the same conditions" [4] while Househ (2013) gave it another religious definition with the focus on Islamic spiritual health "Islamic eHealth is the application and use of information and communication technologies to monitor and support Islamic spiritual health practices with the goal of improving Muslims' spiritual, mental, and physical health status."[5]. WHO realised the value of investing in this field and, thus, called for more studies on eHealth. This was viewed as especially important in countries, such as the Kingdom of Saudi Arabia (KSA), where healthcare outcomes for patients in rural areas are not as good as in urban settings [6].

The Kingdom of Saudi Arabia is a country with one of the largest land masses and populations in the Middle East [7]. It has difficult geographical terrain which makes the delivery of health services challenging. Health services across the country are available from three categories of provider: Ministry of Health $(\mathrm{MOH})$; government run health authority facilities; and the private health sector which is supervised medically by the $\mathrm{MOH}$ to ensure the quality of services [8]. eHealth offers innovative 
solutions for diverse challenges in the management of healthcare services [9]. Healthcare management includes professionals with responsibility for services, resources and partnership in healthcare including eHealth. Drawn from a range of backgrounds, many are clinicians (nurses, physicians, and pharmacists) employed in healthcare management roles [10]. Literature on eHealth status in Saudi Arabia from multiple views has documented a wide range of benefits, such as improving the quality and efficiency of healthcare services, cost reduction, and inter- and intraorganisational communications [11]. However, little is known about the adoption and acceptance of eHealth in Saudi Arabia from these multiple stakeholders perspectives.

In a scoping search, two reviews on eHealth in Saudi Arabia were found $[12,13]$. Both reviews were conducted in 2014 and published in 2016 . The first review was of current literature and had an overall aim of exploring the existing national eHealth programmes, initiatives, and growing efforts in Saudi Arabia [12]. Three main areas focused on were: implementation of eHealth practices, eHealth challenges, and recommendations to enhance eHealth intiatives. The review concluded that the eHealth field is growing in Saudi Arabia even though the number of research publications remains low and limited to few organisations in few geographical areas. More in-depth studies were recommended, especially in the areas of investigating positive and negative aspects of implementing eHealth and understanding the views of different professionals towards eHealth challenges and needs. The second review was a systematic thematic review conducted across all Gulf Cooperation Council (GCC) countries (Bahrain, Kuwait, Oman, Qatar, Saudi Arabia and the United Arab Emirates) [13]. The main aim was to collate all research on eHealth in the GCC to reveal the current state of eHealth research and development in the region. The key themes under investigation were: national benefits from eHealth, implementation and satisfaction with electronic health records, online technologies in medical education, innovative systems, information security and personal health information. The review concluded that Saudi Arabia has a robust medical informatics culture that covers all aspects of eHealth. Two areas were recommended for future 
studies: the cost of eHealth initiatives and religious and gender-related issues in eHealth.

The $\mathrm{MOH}$ in Saudi Arabia has been proactive in adopting technologies to underpin efficiencies in health service delivery under the National Transformation Program 2020 (NTP) which is part of the Saudi Vision 2030 [14]. Notable progress has been made in fields where technology is widely in use, such as statistical applications, communications amongst staff, human resources and integration of health systems under central supervision. With an increasingly active Internet population in Saudi Arabia $(68.5 \%)$ the demand for eHealth services is predicted to continue to grow [15]. The Saudi government has evidenced its intention to continue to support eHealth initiatives that comprehensively focus on advancing the level of technology adoption among all stakeholders [8]. Despite that, the research evidence-base has not kept pace. It has been suggested that this may be due to a lack of qualified IT professionals, poor ICT infrastructure in rural area health facilities and inadequate training programmes [11].

\subsection{Review aim and questions}

The overall aim of this systematic review was to critically appraise, synthesise and present the available evidence on the status of eHealth adoption and acceptance in Saudi Arabia from perspectives of multiple stakeholders. In conducting this review, three questions were proposed:

1. What are the views of health professionals, health IT professionals, and health managers towards eHealth status in Saudi Arabia?

2. What are the factors that influence eHealth adoption and acceptance in Saudi Arabia from the perspectives of health professionals, health IT professionals, and health managers?

3. What are the main facilitators and barriers to implementing eHealth in Saudi Arabia from the perspectives of health professionals, health IT professionals, and health managers? 


\section{Methods}

\subsection{Protocol and search strategy}

Prior to embarking on this systematic review, the Preferred Reporting Items for Systematic review and Meta-Analysis Protocols (PRISMA-P) checklist of 17 items was followed in writing the protocol. PRISMA-P was defined as "a guideline to help authors prepare protocols for planned systematic reviews and meta-analyses that provides them with a minimum set of items to be included in the protocol" [16]. Some benefits such as: improving quality, completeness, and consistency of protocol content as well as increasing awareness of minimum content for protocol reporting were provided to be beneficial for many groups of stakeholders including authors, reviewers, and potential readers [16]. The protocol was registered with the prospective register of systematic reviews (CRD Prospero) [17] and a scoping search was conducted in May 2017.

Five electronic databases were searched: Association for Computing Machinery (ACM), Google Scholar, Medline, ScienceDirect, and Web of Science. These sources were chosen for their reputation of covering the breadth of health, medical and technology articles from scientific and academic journals. The search was restricted to studies conducted in English language as this has been shown to be the primary language for eHealth articles in the GCC region [13]. The search included peer reviewed articles published between January 1993 and May 2017. These dates were selected as 1993 is known to be the year that the first institution was connected to the internet in Saudi Arabia [18]. Table 1 illustrates the inclusion and exclusion criteria to address the review questions.

The following search terms were applied: [eHealth OR e-health OR telemedicine OR telehealth OR telecare or "remote health"] AND ["health professionals" OR "health IT professionals" OR "health managers"] AND [adoption OR acceptance OR facilitators OR barriers] AND [Saudi Arabia]. Titles and abstracts were screened independently by two reviewers and agreement was reached on papers to be excluded with reasons noted. Moreover, an alert was set in all databases for notification of any newly published papers that matched the search criteria. 
Table 1: Inclusion and exclusion criteria

\begin{tabular}{|l|l|}
\hline Participants & $\begin{array}{l}\text { Inclusion: } \\
\text { - Health professionals (medical doctors, nurses, midwives, } \\
\text { pharmacists, dentists, all other allied health professionals e.g. } \\
\text { radiologists and laboratory technicians). } \\
\text { - Health IT professionals. } \\
\text { - Health managers. } \\
\text { Exclusion: } \\
\text { - IT professionals who do not have a role in any health facilities } \\
\text { and organisations }\end{array}$ \\
\hline Interventions & $\begin{array}{l}\text { Inclusion: } \\
\text { The intervention for this study is eHealth. This systematic review } \\
\text { aims to include all published articles and literature around } \\
\text { eHealth adoption, acceptance, facilitators and barriers in Saudi } \\
\text { Arabia from the perspectives of multiple stakeholders. } \\
\text { Exclusion: } \\
\text { Studies that focus on pure technological infrastructure and } \\
\text { products without the users views such as: health technology } \\
\text { applications and Internet of Things (IoT) for health. }\end{array}$ \\
\hline Studies & $\begin{array}{l}\text { Inclusion: } \\
\text { This systematic review focused on peer reviewed primary } \\
\text { published articles and literature with all types of study design } \\
\text { such as quantitative, qualitative and mixed methods. } \\
\text { Exclusion: } \\
\text { Reviews, conference proceedings, blogs, books chapters, and } \\
\text { health website contents were excluded. }\end{array}$ \\
\hline
\end{tabular}

\subsection{Assessment of methodological quality}

Three critical appraisal tools were utilised matching the study design of included articles to minimise the risk of bias by evaluating the methodological quality. Two independent reviewers conducted the quality assessment of the included studies by using the following tools:

1. To assess the quality of the studies with a quantitative design, a survey checklist consisting of twelve questions developed by Crombie (1996) and adopted by the Centre of Evidence Based Management (CEBMa) was used in which every question has to be answered with "yes", "no", or "can't tell" [19]. Questions were presented in short statements in the table for the purpose of clarity. See (Table 2).

2. To assess the quality of the studies with a qualitative design, a qualitative checklist which contains ten questions provided by Critical Appraisal Skills Programme (CASP 2011), Public Health Resource Unit was used in which every question has to be answered with "yes", "no", 
or "can't tell" [20]. Questions were presented in short statements in the table for the purpose of clarity. See (Table 3 ).

3. To assess the quality of the studies with mixed methods design, a quality checklist for mixed methodology studies developed by Mays et al (2001) was used. The checklist contains sixteen items that should be answered with "yes", "no", or "unclear" [21]. See (Table 4). 
Table 2: Critical appraisal tool for eleven quantitative studies based on Crombie (1996) ${ }^{19}$

\begin{tabular}{|c|c|c|c|c|c|c|c|c|c|c|c|}
\hline Criteria & $\begin{array}{l}\text { Bah et al } \\
(2011)^{28}\end{array}$ & $\begin{array}{l}\text { El Mahalli } \\
(2012)^{32}\end{array}$ & $\begin{array}{l}\text { Aldosari } \\
(2014)^{24}\end{array}$ & $\begin{array}{l}\text { Hasanain } \\
\text { \& Cooper } \\
(2014)^{33}\end{array}$ & $\begin{array}{l}\text { El Mahalli } \\
(2015)^{29}\end{array}$ & $\begin{array}{l}\text { Hasanain } \\
\text { et al } \\
(2015)^{34}\end{array}$ & $\begin{array}{l}\text { El Mahalli } \\
(2015)^{31}\end{array}$ & $\begin{array}{l}\text { Almuayqil } \\
\text { et al } \\
(2016)^{26}\end{array}$ & $\begin{array}{l}\text { Jamal } \\
\text { et al } \\
(2016)^{35}\end{array}$ & $\begin{array}{l}\text { El Mahalli } \\
(2016)^{30}\end{array}$ & $\begin{array}{l}\text { Uluc \& } \\
\text { Ferman } \\
(2016)^{36}\end{array}$ \\
\hline $\begin{array}{l}\text { Questions clear and } \\
\text { focused }\end{array}$ & Yes & Yes & Yes & Yes & Yes & Yes & Yes & Yes & Yes & Yes & Yes \\
\hline Design is appropriate & Yes & Yes & Yes & Yes & Yes & Yes & Yes & Yes & Yes & Yes & Unclear \\
\hline $\begin{array}{l}\text { Methods are clearly } \\
\text { described }\end{array}$ & Yes & Yes & Yes & Yes & Yes & Yes & Yes & Yes & Yes & Yes & Yes \\
\hline $\begin{array}{l}\text { Sample introduced } \\
\text { bias }\end{array}$ & Yes & No & Unclear & Yes & No & Yes & No & Unclear & No & No & Unclear \\
\hline $\begin{array}{l}\text { Sample was } \\
\text { representative }\end{array}$ & Yes & Unclear & Unclear & Unclear & Unclear & Unclear & Unclear & Unclear & Yes & Unclear & Unclear \\
\hline $\begin{array}{l}\text { Sample size was } \\
\text { considered }\end{array}$ & Unclear & Yes & Unclear & No & Yes & Yes & Yes & No & No & Yes & Unclear \\
\hline $\begin{array}{l}\text { Response rate was } \\
\text { achieved }\end{array}$ & Unclear & Yes & Unclear & Yes & Yes & Yes & Yes & Yes & Yes & Yes & No \\
\hline $\begin{array}{l}\text { Questionnaire was } \\
\text { Valid and reliable }\end{array}$ & Unclear & Unclear & Yes & Yes & Unclear & Unclear & Unclear & Unclear & Yes & Unclear & Yes \\
\hline $\begin{array}{l}\text { Statistical significance } \\
\text { assessed }\end{array}$ & No & Yes & Yes & No & Yes & Yes & Yes & No & Yes & Yes & Yes \\
\hline $\begin{array}{l}\text { Confidence Intervals } \\
\text { given for main results }\end{array}$ & No & No & No & No & No & No & No & No & No & No & Yes \\
\hline $\begin{array}{l}\text { Confounding factors } \\
\text { accounted }\end{array}$ & Yes & No & No & No & Yes & Yes & No & No & No & No & No \\
\hline $\begin{array}{l}\text { Results were } \\
\text { applicable }\end{array}$ & Yes & Yes & Yes & Yes & Yes & Yes & Yes & Yes & Yes & Yes & Yes \\
\hline
\end{tabular}


Table 3: Critical appraisal for two qualitative studies based on Critical Appraisal Skills Programme (CASP), Public Health Resource Unit ${ }^{20}$

\begin{tabular}{lcc}
\multicolumn{1}{c}{ Criteria } & Alsulame & Alfarra N. \\
et al & $(2016)^{25}$ \\
$(2015)^{27}$ & Yes \\
Statement of aim was clear & Yes & Yes \\
Methodology appropriate & Yes & Can't tell \\
Design appropriate & Yes & Yes \\
Sampling appropriate & Yes & Yes \\
Data collection explained & Yes & Yes \\
Reflexivity & No & No \\
Ethics statement & Yes & No \\
Data analysis & No & Yes \\
Findings discussed & Yes & Yes
\end{tabular}

Table 4: Critical appraisal for two mixed methods studies based on Mays N, Roberts E, Popay J. $(2001)^{21}$

\begin{tabular}{|c|c|c|}
\hline Criteria & $\begin{array}{c}\text { Alasmary } \\
\text { et al } \\
(2014)^{23}\end{array}$ & $\begin{array}{l}\text { Alaboudi } \\
\text { et al } \\
(2016)^{22}\end{array}$ \\
\hline Questions clear and terms defined & Yes & Yes \\
\hline Design was appropriate & Yes & Yes \\
\hline Funding & Yes & Yes \\
\hline Resource system & Yes & Yes \\
\hline Innovation & Yes & Yes \\
\hline Context described & Yes & Yes \\
\hline User system & Yes & Yes \\
\hline Dissemination & Unclear & Unclear \\
\hline Implementation & Unclear & Unclear \\
\hline Sampling generalized & Yes & Unclear \\
\hline Data collection systematic & Yes & Yes \\
\hline Data Analysis systematic & Yes & Yes \\
\hline Results & Yes & Yes \\
\hline Conclusion & Yes & Yes \\
\hline Reflexivity & No & No \\
\hline Ethics & Yes & Yes \\
\hline
\end{tabular}




\subsection{Data extraction}

A Microsoft Word document was created with a separate row for every study and columns for the purpose of extracting eleven key items from the studies: 1) study title, 2) author(s) name, 3) publishing journal, 4) year of publication, 5) study aim, 6) setting(s), 7) methodology, 8) population, 9) intervention, 10) definitions, and 11) key findings. The data were extracted by one reviewer and checked for the accuracy by another (Tables $5 \& 6$ ). 
Table 5: Data extraction

\section{Author (Publishing year)Title/publishing Journal}

Bah et al (2011) Annual survey on the leve and extent of usage of electronic health records in government-related hospitals in Eastern Province, Saudi Arabia/ Perspectives in Health Information Management ${ }^{28}$

El Mahalli et al (2012) Successes and challenges in the implementation and application of telemedicine in the eastern province of Saudi Arabia/ Perspectives in health information management ${ }^{32}$

Alasmary et al (2014) The association between computer literacy and training on clinical productivity and user satisfaction in using the electronic medical record in Saud Arabia/ Journal of medical systems ${ }^{23}$

Hasanain \& Cooper (2014) Solutions to Overcome Technical and Social Barriers to Electronic Health Records Implementation in Saudi Public and Private Hospitals / Journal of Health Informatics in Developing Countries ${ }^{33}$
To determine the level and extent of usage of Electronic Health Records (EHRs) in government-related hospitals in Eastern Province, Saud Arabia

To assess health professionals' perceptions regarding benefits and challenges of telemedicine also willingness to use telemedicine

To investigate the association between computer literacy and training with the clinical productivity and satisfaction of Electronic Medical Records (EMRs)

To investigate the extent of barriers to implementing Electronic Health Records (EHRs) in KSA, particularly social and technical barriers, in order to determine possible solutions to overcome them

\section{Setting}

Eastern

Province,

Saudi Arabia

Eastern

Province,

Saudi Arabia

Riyadh,

Saudi Arabia

study design

using survey and

interviews
Nurses and

physicians

EMRs

Saudi Arabia

Quantitative, cross-sectiona questionnaire

Hospital staff

EHRs (physicians,

nurses,

laboratory

technicians and

scientists,

administrative

staff and

pharmacist) 
Quantitative, based survey
Aldosari, B. (2014) Rates, levels, and determinants of electronic health record system adoption: A study of hospitals in Riyadh, Saudi Arabia/ International journal of medical informatics ${ }^{24}$
To establish the rates, levels, and determinants of EHR Riyadh Saudi hospitals questionnairesystem adoption in a sample of Saudi Arabia

Project

managers

medical

directors, heads

of IT

departments,

and senior

members of the

EHR

development

teams

\section{Senior health}

information

eHealth

eHealth in Saudi Arabia from

the perspective of health

Saudi Arabia Arabia: Current Trends, Challenges and Recommendations/ Enabling Health

informatics professionals

Qualitative, descriptive

professionals

study

El Mahalli A. (2015) Electronic health records Use and barriers among physicians in eastern province of Saudi Arabia/ Saudi Journal of Health Sciences ${ }^{29}$

To assess utilization and Eastern

barriers of EHR system by Province,

physicians at three Saudi Arabia
hospitals

Saudi Arabia

Quantitative,

Physicians

EHRs

cross-sectional

paper-based

questionnaire

design

EHRs software version in Eastern

Province, Saudi Arabia

Hasanain et al (2015) Electronic Medical Record Systems in Saudi Arabia: Knowledge and Preferences of Healthcare Professionals/

Journal of Health Informatics in Developing Countries ${ }^{34}$

El Mahalli A. (2015) Adoption and Barriers to Adoption of Electronic Health Records by Nurses in three Governmental hospitals in Eastern Province, Saudi Arabia/ Perspectives in health information management ${ }^{31}$

To examine both the Jeddah,
knowledge and preferences of Makkah and
current or potential EMR users,
current or potential EMR users, Taif cities, at seven hospitals in three Saudi Arabia cities, within the western region of Saudi Arabia

uantitative,

Health

EMRs cross-sectional, Professionals online and paperbased survey

To assess adoption and Eastern barriers of EHR system by province, nurses at three governmental Saudi Arabia hospitals implementing the same EHR software and functionalities in Eastern province, Saudi Arabia
Quantitative, Nurses

EHRs cross-sectional,

paper-based

survey

functionalities in Eastern
province, Saudi Arabia


Jamal et al (2016) Mobile Phone Use Among Medical Residents: A Cross-Sectional Multi centre Survey in Saudi Arabia/ Journal of Medical Informatics Research ${ }^{35}$

Alaboudi et al (2016) Barriers and challenges in adopting Saudi telemedicine network: The perceptions of decision makers of healthcare facilities in Saudi Arabia/ Journal of Infection and Public Health ${ }^{22}$

Almuayqil et al (2016) Ranking of E-Health Barriers Faced by Saudi Arabian Citizens, Healthcare Professionals and IT Specialists in Saudi Arabia/ Health ${ }^{26}$

El Mahalli et al (2016) Assessment of Pharmacy Information System Performance in Three Hospitals in Eastern Province, Saud Arabia/ Perspectives In Health Information Management ${ }^{30}$

Uluc \& Ferman (2016) A comparative analysis of user insights for e-health development challenges in Turkey, KSA, Egypt \& UAE/ Journal of Management, Marketing \& Logistics $^{36}$
To evaluate the prevalence of mobile phone usage among medical residents and to explore their attitudes, perceptions, and the challenges they experience when using mobile phones in academic and clinical practice

To identify the principle predictive challenges and barriers in adopting and implementing telemedicine in the context of the KSA and investigating the degree of variation within all HCFs sectors, types, and locations

To rank the barriers of e-health in KSA from the perspectives of the Saudi Arabian citizens, healthcare professionals, and IT specialists
Riyadh,

Saudi Arabia

Quantitative,

cross-sectional survey
Medical Residents Mobile phone

Saudi Arabia

Saudi Arabia

To assess the availability and Eastern

usage of pharmacy information Province,

systems (PIS) in three Saudi Arabia

in eastern province,

Saudi Arabia

To assess healthcare Turkey, KSA professionals' insights, for the Egypt \& UAE major challenges of e-health development and a distinctive model and comparative analysis in four emerging countries Turkey, KSA, Egypt and UAE
Three mixed methods

(literature

review,

interviews,

questionnaires

Quantitative, survey based

varied for each

stakeholder

eHealth

Healthcare

Professionals, IT

Specialists

Quantitative, cross-sectional,

paper-based

Decision makers of healthcare facilities

Saudi

Telemedicine

Network

(STN)

survey

\section{Quantitative} explanatory field study with user questionnaires to identify variables

\section{System}

for a model with follow on face-toface interviews to confirm variables
Clinicians,

eHealth professionals, Ministry of Health executives

System
Administrators

exe


Alfarra N. (2016) $A$ qualitative study of an

To obtain insight into the Riyadh

electronic health record: perspective on

planning objectives and implementation at

issues surrounding the

implementation and impact of

King Faisal Specialist Hospital \& Research

Electronic Health Records

(EHRs) at King Faisal Specialist

Journal of Business and Management ${ }^{25}$

Hospital and Research Centre (KFSH\&RC) in Saudi Arabia

Qualitative,

Interpretive

Current patients,
middle/ senior

Phenomenological management, the

Approach using

individual

interviews and

focus groups officer, the chief

operations

officer, the chief

financial officer,

and the chief

medical

information

officer 
Table 6: Data extraction with definitions and findings

\section{Author/Publishing}

\section{year/Title/publishing Journal}

Bah et al (2011) Annual survey on the level and extent of usage of electronic health records in

government-related hospitals in

Eastern Province, Saudi Arabia/

Perspectives in Health Information Management 28

El Mahalli et al (2012) Successes and challenges in the implementation and application of telemedicine in the eastern province of Saudi Arabia/ Perspectives in health information management ${ }^{32}$

\section{Definitions}

EHRs is a longitudinal electronic record of patient health information generated by one or more encounters in any care delivery setting

Telemedicine is: 'the use of medical information exchanged from one site to another via electronic communications to improve patients' health status

\section{Key Findings}

Of 19 hospitals, only three use EHRs all implementing the same systems with core features of laboratory, radiology and pharmacy electronic modules. Main challenges faced by the IT managers in implementing EHRs in their hospitals were related to the uncooperative attitudes of some physicians and nurses toward EHRs

\section{Alasmary et al (2014) The} association between computer literacy and training on clinical productivity and user satisfaction in using the electronic medical record in Saudi Arabia/ Journal of medical systems ${ }^{23}$
EMR defined as an application environment that captures clinical data of patients individually composed with clinical decision support system, computerized order entry and clinical documentation applications
Most frequently cited benefits from adopters were improved quality of care, enhancing access to healthcare and providing patients care and management. Adopters' perceptions were low for other benefits such as easy use of the network, the use of store-and-forward telemedicine and the ability to follow up after face-to-face contacts. The greatest barrier as perceived by health providers was the lack of knowledge about telemedicine
Hasanain \& Cooper (2014) Solutions to Overcome Technical and Social Barriers to Electronic Health Records Implementation in Saudi Public and

\section{None} barrier. Lack of adopting standardized and uniform system was also a barrier. Technical and social barriers were more evident in public hospitals. Inferiority and complexity of EHRs software was raised across 
Private Hospitals / Journal of Health Informatics in Developing Countries ${ }^{33}$ both private and public hospitals. Also, lack of resources such as print paper and ink, lack of HR, training sessions, password access and required skills, lack of sufficient number of computers to be used by the staff and time limits for doctors with numerous patients
Aldosari, B. (2014) Rates, levels, and determinants of electronic health record system adoption: A study of hospitals in Riyadh, Saudi Arabia/ International journal of medical informatics ${ }^{24}$
The term "EHR system" describes the electronic organizational framework and infrastructure that allows EHRs to be stored, accessed, altered, and analysed.
1. Variations exist in the rate and level of EHR system adoption in Saudi Arabia between hospitals (and between regions). There is a need to measure adoption rates and levels in a geographically wider sample. 2 . Further research is needed on the determinants of adoption. The research should include the determinants studied here, and detailed investigations should also be made of physician involvement in the implementation of EHR systems and of user acceptance of the systems.

3. Regarding the implementation phase, an area of weakness across the hospitals involves the legacy of paper data systems, including document scanning, record management, and data conversion. These deficiencies need to be addressed so that the efficiency and usefulness of EHR systems can be maximized in adopting hospitals, and to ease implementation by current non adopters.

4. In the maintenance phase, there is a weakness with respect to software updating and maintenance. The reasons for this weakness need to be identified.

5. For the improvement phase, there is a deficiency in health information communication and sharing, including deficiencies in the development of data repositories, in the establishment of information networks, and in information exchange. The barriers to information sharing need to be better defined, including the problem of interoperability between the many different hospital EHR systems in use
Alsulame et al (2015) eHealth in Saudi Arabia: Current Trends, Challenges and Recommendations/ Enabling Health Informatics Applications ${ }^{27}$

\section{None}

Challenges were grouped as: 1) Organizational and Behavioural

2) Technological and Professional; and, 3) Privacy and Confidentiality 
El Mahalli A. (2015) Electronic health None records: Use and barriers among physicians in eastern province of Saudi Arabia/ Saudi Journal of Health Sciences $^{29}$

Hasanain et al (2015) Electronic Medical Record Systems in Saudi

Arabia: Knowledge and Preferences of Healthcare Professionals/ Journa of Health Informatics in Developing Countries $^{34}$
None
There was low adoption of chart review functionality with users reporting 'system hanging up problem' and additional time for data entry affecting utilization in all 3 hospitals. Problems were reported with drug alert systems. Lab, radiology and pharmacy order entry rates were high. Communication tools were not in use for patient contact and in limited use in hospital due to lack of internet access in hospitals. Loss of access to medical records was cited as an issue caused by power failure/computer crashes. Training and support were lacking. Confidentiality, security and privacy were noted barriers to EHR adoption

As computer literacy levels increase so too do staff preferences for EMR systems. Hospitals need to offer English language and computer literacy training to increase staff acceptance of the EMR system

El Mahalli A. (2015) Adoption and Barriers to Adoption of Electronic Health Records by Nurses in three Governmental hospitals in Eastern Province, Saudi Arabia. Perspectives in health information management ${ }^{31}$
Health Information Technology (HIS) is: 'the application of information processing involving both computer hardware and software that deals with the storage, retrieval, sharing, and use of health care information, data, and knowledge for communication and decision making'
Under-utilization of most EHRs functionalities. No utilization of any communication tools with patients. Most frequently cited barrier among all hospitals was 'loss of access to medical records transiently if computer crashes or power fails'. Also lack of training and support, additional time for data entry and 'system hanging up problems', complexity and lack of customisability of systems 
Jamal et al (2016) Mobile Phone Use Among Medical Residents: A Cross-

Sectional Multicenter Survey in Saudi Arabia/ Journal of Medical

Informatics Research ${ }^{35}$
None

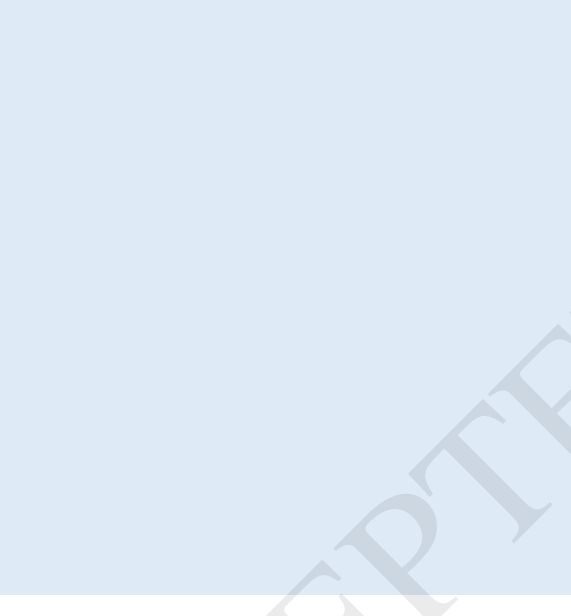

Alaboudi et al (2016) Barriers and challenges in adopting Saudi

telemedicine network: The

perceptions of decision makers of

healthcare facilities in Saudi Arabia/

Journal of Infection and Public Health 22
UTAUT: Unified Theory of Acceptance and Use of Technology

TOE: Technology-OrganisationEnvironment

ETSSM: Evaluating Telemedicine Systems Success Model

HCF: Health Care Facilities
Adoption of mobile phone usage was found to be $99.0 \%$. Negative correlation between age of participants and duration of mobile phone use. Apple iPhone iOS was predominant in medical population. English most commonly used on mobile phones despite native language being Arabic. WhatsApp and phone calls were the most commonly used tools. Medical communication, drug and medical references and medical calculation applications were the most commonly used. Technically, short battery life was the main issue, and distraction at least once per week. All participants agreed with integrating medical staff mobile phones with hospital information systems. Most participants described themselves as self-learners, half learned from peers and a quarter from the internet. Only $6.9 \%(n=7 / 101)$ had received any formal training on the medical use of mobile phones. Over half of participating residents thought it was safe to discuss patients over their personal, nonencrypted email
Almuayqil et al (2016) Ranking of E- None

Health Barriers Faced by Saudi

Arabian Citizens, Healthcare
Citizens ranked the connectivity of information system as the top barrier with cultural barriers least barrier. Healthcare professionals ranked connectivity as the top barrier and technical expertise/computer 
Professionals and IT Specialists in Saudi Arabia/ Health ${ }^{26}$ skills as least. The top ranked barrier from the perspective of IT Specialists was medication safety with security and privacy least

El Mahalli et al (2016) Assessment of Pharmacy Information System

Performance in Three Hospitals in Eastern Province, Saudi Arabia/ Perspectives In Health Information Management ${ }^{30}$

Uluc \& Ferman (2016) A comparative analysis of user insights for e-health development challenges in Turkey, KSA, Egypt \& UAE/ Journal of Management, Marketing \& Logistics ${ }^{36}$
PIS is a system that can help pharmacists 'reduce clinical errors with intelligent warnings,

messages, and rejection notices; gain immediate access to clinical information from throughout the enterprise; access all relevant data from a single centralized processing screen; and minimise lots revenue with the option to charge on administration
PIS include computerized provider order entry and clinical decision support, integrated with EHR, prescribing and transcription functionality. Dispensing remains a manual process. Barcode-assisted medication administration systems are not in use to verify patient identify nor to electronically check dose administration. Computerized adverse drug event monitoring was not linked to EHR
E-Health 'refers to the use of information technologies in healthcare services. It has a wide scope covering many concepts such as tele-health, mobile health, use of EHR, consumer health IT data and big data in digital health systems
ICT infrastructure, regulations, cultural and clinical adaptation of users, financing, supply chain management are some major challenges.

Specially trust to e-business in healthcare, compliant use of big data in digital health and patient privacy play a key role for faster development of e-health. An original framework of a model for assessing the major challenges of e-health development in emerging countries was produced 
Alfarra N. (2016) A qualitative study of an electronic health record:

perspective on planning objectives

and implementation at King Faisal

Specialist Hospital \& Research Centre

(KFSH \& RC), Saudi Arabia/ IOSR

Journal of Business and

Management ${ }^{25}$
Three categories of impacts were identified according to who was

affected. These related to the healthcare providers, the patients and the $\mathrm{KFSH} \& \mathrm{RC}$ respectively. The impact on the healthcare providers included increased convenience and efficiency in data entry, retrieval, storage and distribution; access to the EHR system; information and knowledge growth; empowering the staff; and impacts on healthcare providers attitude toward using the EHR system. The impact on patients was mainly felt in terms of the quality of care and the communication flow between the patients and healthcare providers. The KFSH \& RC was affected by providing a better work environment to its employees by reducing the number of paper files stored; the educational benefits and learning experiences gained; and improved communication between staff members and patients while increasing their ability to control the quality of care. The positive impacts of the EHR implementation far outweigh the negative impacts. Therefore, it is logical to conclude that the benefits of the EHR systems are outweighed their negative impacts. In descending order, the most frequently

mentioned benefits are: quick data retrieval, easy and quick data input, easy access to KFSH \& RC EHRs, facilitating smooth communication with external healthcare providers, enhancing the flow of information about patients, facilitating communication among staff members improving the format of records, and increasing patient safety 


\subsection{Data synthesis}

Data pooled from the studies were presented narratively in tables. Findings were then considered with a focus on factors that may influence adoption and acceptance of eHealth from multiple stakeholder perspectives.

\section{Results}

\subsection{Study Characteristics}

The result of the searches returned 176 papers of which 110 papers were included after running a duplication removal process. Title screening reduced the total further, then 45 abstracts were screened followed by 25 full texts. These were assessed for eligibility and finally 15 papers [2236] were included for meeting all criteria of inclusion and exclusion (Figure 1) [37]. 


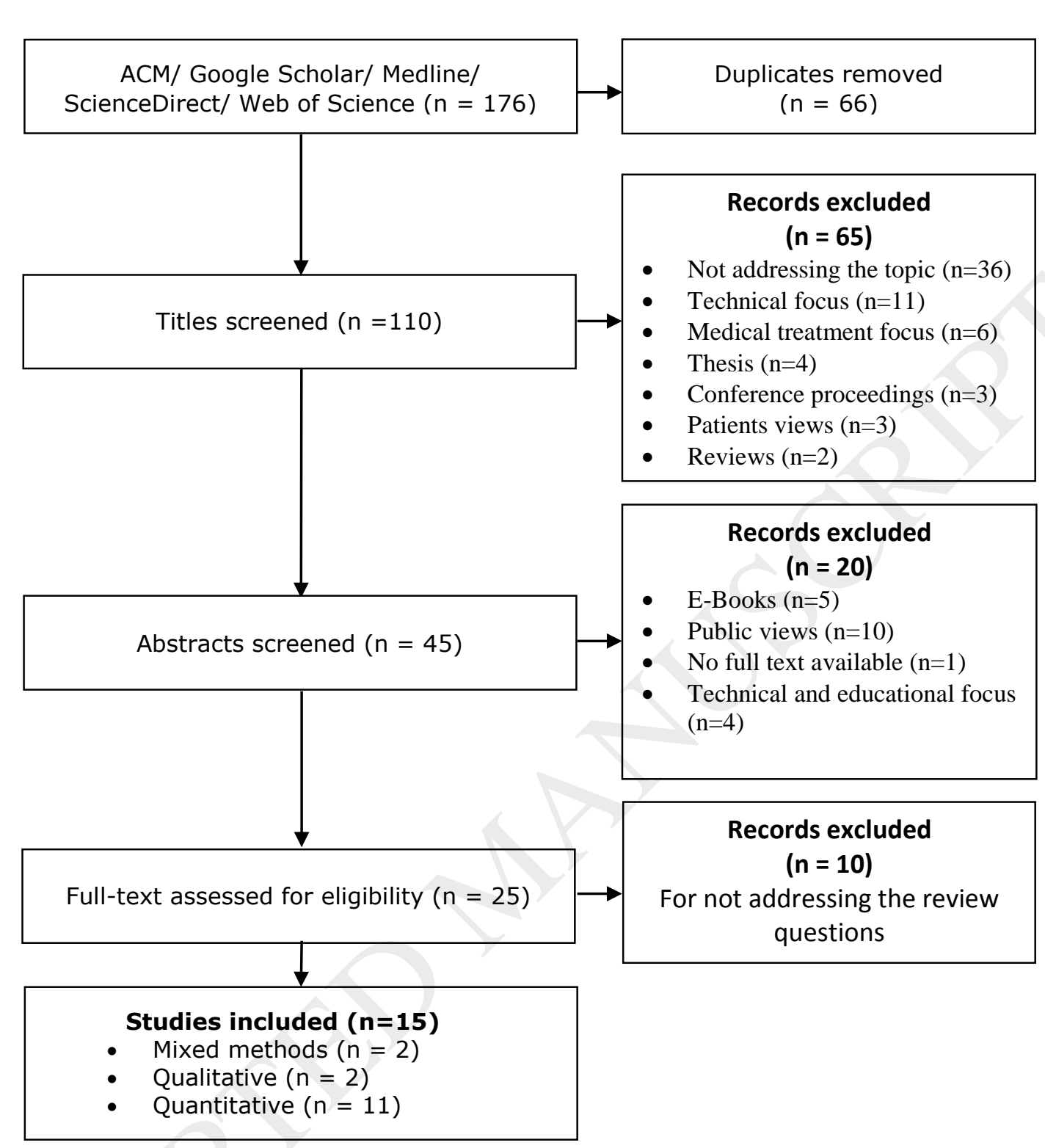

Figure 1: PRISMA flow diagram ${ }^{37}$

All included studies were published between 2011 and 2016. One study was published in 2011 [28], one study in 2012 [32], three studies in 2014 [23,24,33], four studies in 2015 [27,29,31,34] and six studies in 2016 $[22,25,26,30,35,36]$. In regards to the geographical setting, one study was comparative of eHealth in four countries (Turkey, Saudi Arabia, Egypt and UAE) [36]. The remaining 14 studies were conducted in Saudi Arabia with 1 study in Makkah region [34], 4 studies in Riyadh city which is the capital of Saudi Arabia [23-25,35], 5 studies were conducted in the 
Eastern province of Saudi Arabia [28-32], and finally 4 studies did not specify any specific geographical location within Saudi Arabia $[22,26,27,33]$.

In regards to the methodology and methods, 11 studies were quantitative in design [24,26,28-36]. Four of these studies used paper-based crosssectional questionnaires [29-32], two studies used online survey [28,35], one study used mixed online and paper-based surveys [34], while four studies did not supply information on the type of questionnaire in use in their studies $[24,26,33,36]$. Two studies were qualitative and used interview and focus group approaches $[25,27]$ and the remaining two studies were mixed methods using a combination of questionnaires and interviews for collecting data $[22,23]$.

In terms of intervention, six studies discussed Electronic Health Records (EHRs) $[24,25,28,29,31,33]$, three studies were about eHealth in general $[26,27,36]$ while two studies specifically investigated Electronic Medical Records (EMRs) in hospitals [23,34]. Other studies examined different interventions, such as, Pharmacy Information System [30], use of mobile phones in health [35], telemedicine [32], and the Saudi Telemedicine Network (STN) [22].

With regards to population, participants were described differently in each study. Health professionals were the target participants of six studies $[23,29,31,32,34,35]$. Health IT managers were the focus in two studies $[27,28]$ while senior and middle level health managers participated in one study [25]. The remaining studies targeted mixed and random participants of the three groups of professionals matching the inclusion criteria $[22,24,26,30,33,36]$.

With reference to the study aims, five studies identified barriers of intervention implementation with the focus on challenges and adoption level $[26,29,31,33,36]$, two studies assessed the perceptions of health professionals [22,32], two studies evaluated the availability and prevalence of the interventions under study $[30,35]$, the remaining six studies had a variety of aims within the main scope of the review [23- 
$25,27,28,34]$. With regards to the intervention definitions, only eight studies provided definitions for the focus of the study [22-24,28,30$32,36]$.

In terms of the quality, all studies included were of good quality in regard to the clarity of aims and questions. All studies appropriately described their methods in use, however, in some studies there was a need for better identification of whether the undertaken design was appropriate for conducting the study. Different sample sizes were identified in each study, however, two-third of the studies (10 out of 15 ) $[22,24,26,29-34,36]$ did not clarify whether the sample selected was considered to be representative or not. The same lack of clarity applied to bias introduction in quantitative design studies as 8 studies out of 11 did not propose how bias would be dealt with $[24,26,29-32,35,36]$. Only one study considered technology acceptance theories to underpin their research which was about barriers and challenges in adopting Saudi Telemedicine Network [22]. In this sole study, three models were used: the Unified Theory of Acceptance and Use of Technology (UTAUT), the Technology Organisation Environment (TOE) theoretical framework, and the Evaluating Telemedicine Systems Success Model (ETSSM). The study concluded that the top three barriers to adoption and implementation of telemedicine by the healthcare facilities (HCF) decision makers are: (i) the availability of adequate sustainable financial support to implement, operate, and maintain the telemedicine system, (ii) ensuring conformity of telemedicine services with core mission, vision, needs and constraints of the HCF, and (iii) the reimbursement for telemedicine services. These findings were based on the response of a representative sample of 905 participants and the barriers were highlighted as most significant to Saudi Arabian context. However, that contradicts the findings from other studies of the same context that presented lack of technical and professional training sessions and confidentiality, security and data privacy issues at higher significance than financial barriers $[29,31,33]$. 


\section{2. eHealth influencing factors identified}

From the 15 studies included, 39 factors were identified as influences affecting the adoption and acceptance of eHealth in Saudi Arabia from the perspective of multiple stakeholders. For the purpose of clarity in this systematic review, all factors were grouped into six clusters based on their nature (Figure 2). 


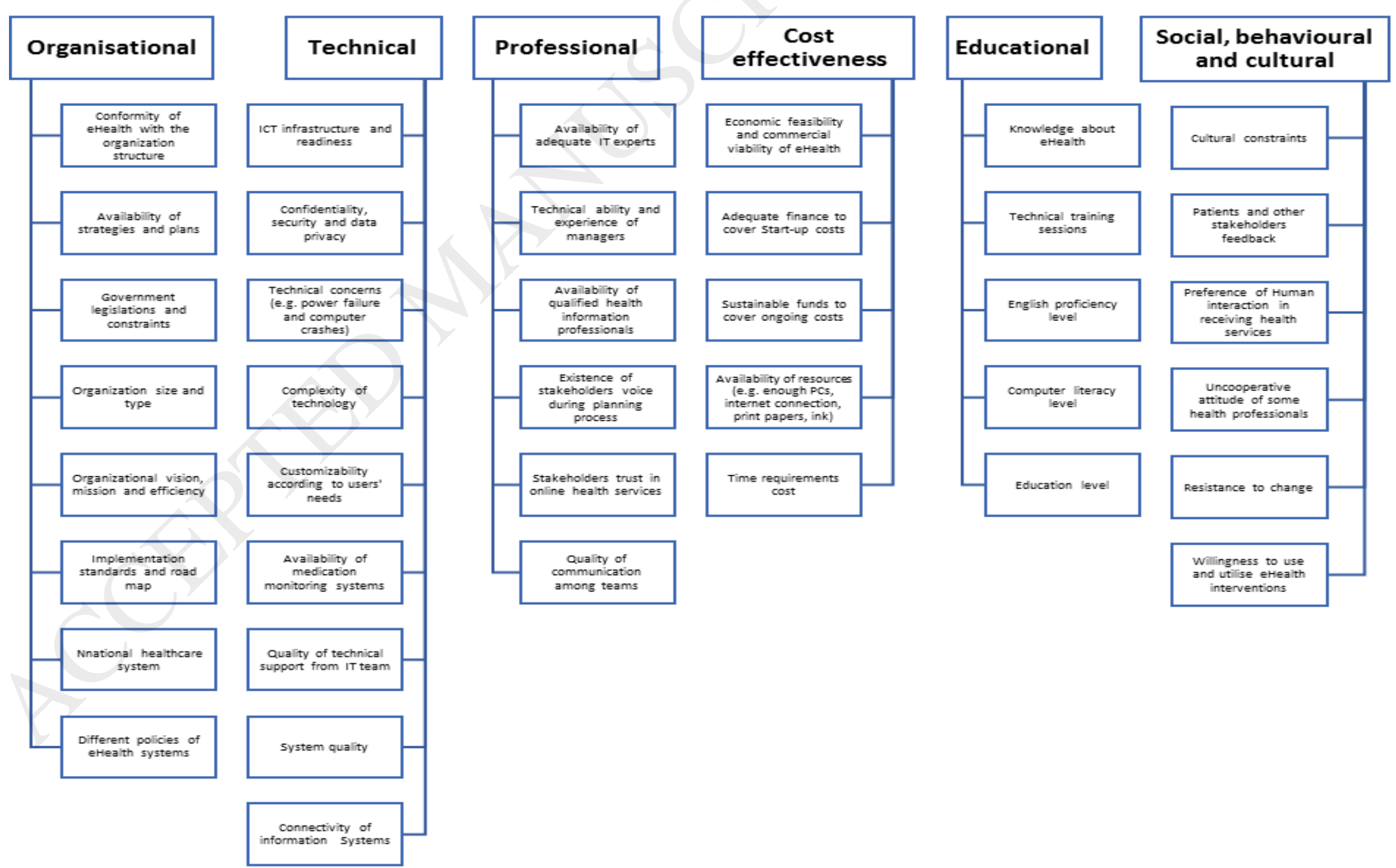

Figure 2: Clusters of factors that influence eHealth adoption and acceptance in Saudi Arabia 
Further, the factors are described based on the 15 studies included.

Organisational factors which are related to the healthcare organisations and facilities. These factors vary from one organisation to another based on the level of bureaucracy and the clarity of policies and procedures within the work place. Literature from the studies has shown evidence on how these factors play a role in affecting the acceptance of technology by the health professionals and managers.

Technical factors which refer to the usage, processes and operations that are involved with technical aspects such as IT infrastructure, eHealth applications, and information security. These factors were reported to be major challenges requiring large budget allocation to cover operations and maintenance.

Professional factors these emphasised the importance of having adequate numbers of qualified professionals in the organisation with both the technical background to support systems and health background to run health technology systems with efficiency.

Cost effectiveness factors showed how financial support may affect both the adoption level of health organisations and the acceptance level by the professionals. Adequate finance to cover start-up costs, ongoing costs, and secure the sustainability fund to work on providing enough resources were all considered success signs of eHealth adoption and acceptance. Time can also be considered as a cost effectiveness factor required to front load implementation, adoption and acceptance of new innovations and ways of working.

Educational factors may influence an individual's attitude towards technology adoption and acceptance through their personal experience. These factors were mentioned in the studies in different ways such as: level of education, lack of training, English language proficiency level and computer or digital literacy.

Social, behavioural and cultural factors in which the level of the adoption can be challenged by the social and cultural beliefs of the stakeholders. Resistance to change, willingness to utilise technology and 
preference for human or computer interaction in receiving healthcare services may influence adoption and acceptance levels.

\section{Discussion}

This review sets out the available evidence of the adoption and acceptance of eHealth in Saudi Arabia from the perspective of multiple stakeholders. Despite the issues raised, the field of eHealth showed evidence of continual growth in the country in both publications and awareness of significance. However, there has been a lack of studies that focus on the perspective of health management professionals. In general, the findings showed consistency with previous studies such as the study conducted by Altuwajiri in 2008 which emphasised four major groups of barriers to eHealth in Saudi Arabia: economic, technological, organisational, and behavioural barriers [11].

A new study published in 2018 was picked out by notification alert that was set on searched databases [38]. It was carried out in three $\mathrm{MOH}$ hospitals in Makkah city, KSA and aimed to: 1) Assess the utilization status of eHealth in Makkah city hospitals, 2) Measure the usefulness of eHealth in delivering good healthcare in Saudi Arabia, and 3) Find out the challenges / barriers in implementing eHealth services in Saudi Arabia. In this study, a questionnaire was used to collect data from a sample size of 51 administrative and medical staff. The study found out that apart from the shortage of operational resources such as computers and the staff technical ability, cost and expertise in innovative systems in IT were the main challenges. These findings showed similarity with the results that were pooled from the studies included in this review.

In 2011, the MOH launched a national eHealth strategy in order to achieve its innovative vision for eHealth "safe, efficient health system, based on the care centered on a patient, standard-oriented, and supported by the eHealth" [9]. This ambitious strategy anticipated that eHealth would bring huge benefits for patients, providers, and health system managers. A roadmap of implementation was established to track progress within the process which was planned to be carried out in two phases ( 5 years each). Furthermore, it has been found that the governance model for the Saudi 
NTP 2020 [14] has set five phases to achieve the objectives for all government bodies concerned including the $\mathrm{MOH}$. These phases progress gradually from first (i) identifying the challenges, moving to the second, (ii) developing initiatives and plans, followed by third, (iii) implementing plans, then fourth, (iv) publishing outcomes, to finally the fifth phase which concentrates on ( $v$ ) auditing, improving and adding new initiatives [14]. The $\mathrm{MOH}$ has to achieve 15 objectives as part of meeting the Saudi NTP 2020. The third objective of the MOH plan is to improve the efficiency and effectiveness of the healthcare sector through the use of IT and digital transformation [14].

Findings from this review may help key professionals assigned to work on achieving the $3^{\text {rd }}$ objective to determine the $1^{\text {st }}$ phase of operation which focuses on identifying current challenges. This study, in addition, complements the previously identified factors thought to influence the adoption and acceptance of eHealth in Saudi Arabia and shall address the current challenges and barriers to help with prioritising the main areas for improvement. However, the similarities and differences between the findings of this systematic review and the extent to which they apply to all or parts of Saudi Arabia have yet to be established.

\subsection{Strengths and Weaknesses}

Strengths of this review include following best practice such as the Preferred Reporting Items for Systematic Reviews and Meta-Analysis Protocols (PRISMA-P) for writing the protocol [16] and PRISMA checklist for reporting the results [37]. Another strength is that the review was conducted by a multidisciplinary team acting as independent reviewers at each stage to minimise the risk of bias [39]. However, there were weaknesses that could limit the transferability of the findings and recommendations such as: the limited number of studies and geographical scale which makes it difficult for findings and recommendations to be generalised, therefore, we suggest applying caution upon interpretation. 


\section{Conclusion}

This review has highlighted the status of eHealth research in Saudi Arabia from the perspective of multiple stakeholders and identified some of the main barriers and challenges that influence the adoption and acceptance of eHealth. Thirty-nine factors in six clusters were identified that influence eHealth adoption and acceptance in the country. Although the number of eHealth publications is increasing, there remains the need to investigate the views of specific stakeholder groups towards eHealth, taking into account their voices during the planning process of any future projects. Finally, due to the limitation of eHealth studies to certain regions, it is recommended to extend research into the experience and extent of eHealth adoption and acceptance levels in different geographical settings across the country to draw a clearer picture of the current practice and future plans for eHealth.

\section{Summary points}

What was known about eHealth in Saudi Arabia:

- Literature on eHealth status in Saudi Arabia has documented a wide range of benefits, such as improving the quality and efficiency of healthcare services, cost reduction, and inter- and intra-organisational communications

- The eHealth field is growing in Saudi Arabia even though the number of research publications is limited to few organisations in few geographical areas

- In 2011, the Ministry of Health (MOH) launched a national eHealth strategy

- Little was known about the adoption and acceptance of eHealth in Saudi Arabia from multiple stakeholders perspectives

What this systematic review has added to the knowledge:

- Thirty-nine factors were identified as influences affecting the adoption and acceptance of eHealth in Saudi Arabia from the perspective of multiple stakeholders

- There remains a need to investigate the views of specific stakeholder groups towards eHealth 
- Findings from this review may help key professionals to address the current challenges and barriers and so prioritise the main areas for improvement

- This review recommends further research into the experience and extent of eHealth adoption and acceptance levels in different geographical settings across the country to draw a clearer picture of the current practice and future plans for eHealth in Saudi Arabia

\section{Conflict of interest}

None declared

\section{Funding}

This systematic review was conducted as a first phase of doctoral study sponsored by the Ministry of Health, Saudi Arabia.

\section{AUTHOR STATEMENT}

Manuscript title: A systematic review of the adoption and acceptance of eHealth in Saudi Arabia: views of multiple stakeholders

Corresponding author:

Dr Katie MacLure

School of Pharmacy \& Life Sciences

Robert Gordon University

Sir Ian Wood Building, Garthdee Road, Aberdeen UK, AB10 7GJ

Email: K.M.MacLure@rgu.ac.uk

\begin{tabular}{|l|l|}
\hline \multicolumn{1}{|c|}{ Authors name } & \multicolumn{1}{c|}{ Contribution to the work } \\
\hline $\begin{array}{l}\text { Abdullah Alshahrani (AA), } \\
\text { Professor Derek Stewart (DS), and } \\
\text { Dr Katie MacLure (KM) }\end{array}$ & $\begin{array}{l}\text { AA wrote the review protocol with } \\
\text { support from KM and DS. }\end{array}$ \\
& $\begin{array}{l}\text { AA registered the protocol with the } \\
\text { prospective register of systematic } \\
\text { reviews (CRD Prospero) with } \\
\text { support from KM }\end{array}$ \\
& $\begin{array}{l}\text { - AA performed the database searches } \\
\text { - AA and KM conducted the quality } \\
\text { assessment of the included studies }\end{array}$ \\
\hline
\end{tabular}




\begin{tabular}{|l|l|}
\hline - & $\begin{array}{l}\text { Data was extracted by AA and } \\
\text { checked for the accuracy by KM } \\
\text { - AA performed the data synthesis and } \\
\text { discussion with support from KM }\end{array}$ \\
- & DS added to the discussion of the \\
review & AA designed Word documents, \\
- & tables, and figures. \\
- AA wrote the first draft of the \\
manuscript with KM support. \\
DS and KM commented on the draft \\
which was updated by AA. \\
KM finalised the manuscript pre- \\
submission for AA to upload.
\end{tabular}

\section{References}

[1] Oh H, Rizo C, Enkin M, Jadad A. What is eHealth (3): a systematic review of published definitions. J Med Internet Res 2005; 7(1), pp. e1.

[2] Pagliari C, Sloan D, Gregor P, Sullivan F, Detmer D, Kahan JP, Oortwijn W, MacGillivray S. What is eHealth (4): a scoping exercise to map the field. Journal of medical Internet research. 2005 Jan;7(1).

[3] World Health Organization. Strategy 2004-2007. (2004). eHealth for health care delivery. Available at: http://www.who.int/ehealth/about/en/ accessed 15 October 2017

[4] Pretlow R. eHealth International: A Cutting Edge Company for a New Age in Health Care. Available

at: http://www.ehealthnurse.com/ehealthi.html accessed in 15 June 2017

[5] Househ MS. Islamic E-health: definitions, applications and challenges. InITCH 2013 (pp. 281-285).

[6] Al-Shorbaji N. E-Health in the Eastern Mediterranean region: A decade of challenges and achievements. East Medit Heal J 2008; 14(S1), pp. A157A157.

[7] World Atlas. Countries, Saudi Arabia. (2016). Available at:

http://www.worldatlas.com/webimage/countrys/asia/sa accessed 13

September 2017

[8] General Authority for Statistics. Health Statistics. (2016). Available at: http://www.stats.gov.sa/statistics/health accessed 21 August 2017 
[9] Ministry of Health. National e-health strategy. (2011). Available at: https://www.moh.gov.sa/en/Ministry/nehs/Pages/default.aspx accessed 12 October 2017

[10] Egger D, Ollier E, Tumusiime P, Bataringaya J. Strengthening management in low-income countries. World Health Organization 2005. [11] Altuwaijri M. Electronic-health in Saudi Arabia. Just around the corner? Saud med J 2008; 29(2), pp. 171-178.

[12] Alsulame K, Khalifa M and Househ M. E-Health status in Saudi Arabia: A review of current literature. Heal Pol and Techno J 2016; 5(2), pp. 204210.

[13] Weber A, Turjoman R, Shaheen $Y$, et al. Systematic thematic review of e-health research in the Gulf Cooperation Council (Arabian Gulf): Bahrain, Kuwait, Oman, Qatar, Saudi Arabia and United Arab Emirates. J of telemed and teleca 2016, 23(4), pp. 452-459.

[14] Saudi Vision 2030. (2016). The National Transformation Program 2020 (NTP). Available at: http://vision2030.gov.sa/en/node/125 accessed 12 June 2017

[15] Communication and Information Technology Commission. (2015). CITC annual report. Available at:

http://www.citc.gov.sa/en/mediacenter/annualreport/Documents/PR REP 011Eng.pdf accessed 28 July 2017

[16] Moher D, Shamseer L, Clarke M, et al. Preferred reporting items for systematic review and meta-analysis protocols (PRISMA-P). Systematic reviews 2015; 4(1), pp. 1.

[17] Alshahrani A, MacLure K, Stewart D. Status of eHealth research in Saudi Arabia: a systematic review protocol. (2017). Available at:

http://www.crd.york.ac.uk/PROSPERO/display record.php?ID=CRD42017 065009 accessed 1 May 2017

[18] Al-Tawil K. The internet in Saudi Arabia. King Fahad University of Petroleum \& Minerals 1999.

[19] Crombie IK. The pocket guide to critical appraisal: a handbook for health care professionals, 1997.

[20] Critical Appraisal Skills Programme (CASP). (2013). CASP systematic review checklist. Available at: http://www.casp-uk.net/casp-toolschecklists accessed 12 September 2017 
[21] Mays N, Roberts E and Popay J. Synthesising research evidence. Studying the organisation and delivery of health services: Research methods. (2001) pp. 188-220. Available at:

http://www.refworks.com/refworks2/default.aspx?r=file: :get file\&file na $\underline{\text { me }=\text { RW } 29}$ accessed 13/09/2017

[22] Alaboudi A, Atkins A, Sharp B, et al. Barriers and challenges in adopting Saudi telemedicine network: The perceptions of decision makers of healthcare facilities in Saudi Arabia. J of Infec and Pub Heal 2016; 9(6), pp. $725-733$.

[23] Alasmary M, El Metwally A and Househ $M$. The association between computer literacy and training on clinical productivity and user satisfaction in using the electronic medical record in Saudi Arabia. J of med syst 2014; 38(8), pp. 69-69.

[24] Aldosari B. Rates, levels, and determinants of electronic health record system adoption: A study of hospitals in Riyadh, Saudi Arabia. International J of med inform 2014; 83(5), pp. 330-342

[25] Alfarra N. A qualitative study of an electronic health record: perspective on planning objectives and implementation at King Faisal Specialist Hospital \& Research Centre (KFSH \& RC), Saudi Arabia. IOSR J of Bus and Manag 2016.

[26] Almuayqil S, Atkins AS and Sharp B. Ranking of E-Health Barriers Faced by Saudi Arabian Citizens, Healthcare Professionals and IT Specialists in Saudi Arabia. Health 2016; 8(10), pp. 1004.

[27] Alsulame K, Khalifa M and Househ M. eHealth in Saudi Arabia: Current Trends, Challenges and Recommendations. Stud Heal Technol Inform 2015; 213, pp. 233-236.

[28] Bah S, Alharthi H, El Mahalli A, et al. Annual survey on the level and extent of usage of electronic health records in government-related hospitals in Eastern Province, Saudi Arabia. Perspect Heal Inf Manag 2011; 8(Fall), pp. 1b.

[29] El Mahalli AA. Electronic health records: Use and barriers among physicians in eastern province of Saudi Arabia. Saud J of Heal Sci 2015.

[30] El Mahalli A, El-Khafif S and Yamani W. Assessment of Pharmacy Information System Performance in Three Hospitals in Eastern Province, Saudi Arabia. Perspect in Heal Inform Manag 2016; 13, pp. 1b-1b. 
[31] El Mahalli A. Adoption and barriers to adoption of Electronic Health Records by nurses in three governmental hospitals in Eastern Province, Saudi Arabia. Perspect in heal inform manag 2015; 12, pp. 1 f.

[32] El Mahalli AA, El-Khafif SH and Al-Qahtani MF. Successes and challenges in the implementation and application of telemedicine in the eastern province of Saudi Arabia. Perspect in heal inform manag 2012; 9, pp. 1-27.

[33] Hasanain RA and Cooper H. Solutions to overcome technical and social barriers to electronic health records implementation in Saudi public and private hospitals. J of Heal Inform in Devel Count 2014; 8(1).

[34] Hasanain RA, Vallmuur $\mathrm{K}$ and Clark M. Electronic medical record systems in Saudi Arabia: knowledge and preferences of healthcare professionals. J of Heal Inform in Devel Count 2015; 9(1).

[35] Jamal A, Temsah M, Khan S, et al. Mobile Phone Use Among Medical Residents: A Cross-Sectional Multicenter Survey in Saudi Arabia. J of Med Inform Res 2016; 4(2), pp. e61-e61.

[36] Uluc CI and Ferman M. A comparative analysis of user insights for ehealth development challenges in Turkey, Kingdom of Saudi Arabia, Egypt and United Arab Emirates. J of Manag Market and Logist 2016; 3(2).

[37] Moher D, Liberate A, Tetzlaff J, Altman D, PRISMA Group. Preferred reporting items for systematic reviews and meta-analyses: the PRISMA statement. PLoS medici 2009; 6(7), pp. e1000097.

[38] Zaman T, Abdulraheem T, Alharbi G, et al. E-Health and its transformation of healthcare delivery system in Makkah, Saudi Arabia. Inter J of Med Res \& Heal Sci 2018; 7(5), 76-82.

[39] MacLure K, Paudyal V , Stewart D. Reviewing the literature, how systematic is systematic? Internat J of Clin Pharma 2016; 38:695-694. doi:10.1007/s11096-016-0288-3 